\title{
Simple Model of Complete Precessing Black-Hole-Binary Gravitational Waveforms
}

\author{
Mark Hannam, ${ }^{1}$ Patricia Schmidt, ${ }^{1,2,3}$ Alejandro Bohé, ${ }^{4,5}$ Leïla Haegel, ${ }^{4,6}$ Sascha Husa, ${ }^{4}$ \\ Frank Ohme, ${ }^{1}$ Geraint Pratten, ${ }^{1,7}$ and Michael Pürrer ${ }^{1}$ \\ ${ }^{1}$ School of Physics and Astronomy, Cardiff University, Queens Building, CF24 3AA Cardiff, United Kingdom \\ ${ }^{2}$ LIGO Laboratory, California Institute of Technology, MS 100-36, Pasadena, California 91125, USA \\ ${ }^{3}$ Theoretical Astrophysics 350-17, California Institute of Technology, Pasadena, California 91125, USA \\ ${ }^{4}$ Departament de Física, Universitat de les Illes Balears, Carretera Valldemossa, E-07122 Palma, Spain \\ ${ }^{5}$ Max-Planck-Institut für Gravitationsphysik, Albert-Einstein-Institut Am Mühlenberg 1, 14476 Golm, Germany \\ ${ }^{6}$ DPNC, Université de Genève, Ecole de Physique, 24 Quai Ernest Ansermet, 1202 Genève, Switzerland \\ ${ }^{7}$ Astronomy Centre, University of Sussex, Brighton BN1 9QH, United Kingdom
}

(Received 19 August 2014; published 7 October 2014)

\begin{abstract}
The construction of a model of the gravitational-wave (GW) signal from generic configurations of spinning-black-hole binaries, through inspiral, merger, and ringdown, is one of the most pressing theoretical problems in the buildup to the era of GW astronomy. We present the first such model in the frequency domain, PhenomP, which captures the basic phenomenology of the seven-dimensional parameter space of binary configurations with only three key physical parameters. Two of these (the binary's mass ratio and an effective total spin parallel to the orbital angular momentum, which determines the inspiral rate) define an underlying nonprecessing-binary model. The nonprecessing-binary waveforms are then twisted up with approximate expressions for the precessional motion, which require only one additional physical parameter, an effective precession spin, $\chi_{p}$. All other parameters (total mass, sky location, orientation and polarization, and initial phase) can be specified trivially. The model is constructed in the frequency domain, which will be essential for efficient GW searches and source measurements. We have tested the model's fidelity for GW applications by comparison against hybrid post-Newtoniannumerical-relativity waveforms at a variety of configurations-although we did not use these numerical simulations in the construction of the model. Our model can be used to develop GW searches, to study the implications for astrophysical measurements, and as a simple conceptual framework to form the basis of generic-binary waveform modeling in the advanced-detector era.
\end{abstract}

Introduction.-The imminent commissioning of secondgeneration laser-interferometric gravitational-wave detectors will bring us closer to the era of gravitational-wave (GW) astronomy, which carries the potential to revolutionize our understanding of astrophysics, fundamental physics, and cosmology [1]. Among the most promising GW sources are the inspiral and merger of black-hole binaries. Detection and interpretation of these signals requires analytic models that capture the phenomenology of all likely binary configurations; most of these will include complex precession effects due to the black-hole spins. However, most of the current models of the two black holes' inspiral, their merger, and the ringdown of the final black hole, consider only configurations where the blackhole spins are aligned with the binary's orbital angular momentum, which do not involve any precession.

The binary's early inspiral can be modeled with analytic post-Newtonian (PN) calculations, but the late inspiral and merger require 3D numerical solutions of the full nonlinear Einstein equations. These expensive numerical relativity (NR) calculations must span a parameter space of binary configurations that covers, for noneccentric inspiral, seven dimensions: the mass ratio of the binary, and the components of each black hole's spin vector; the total mass of the system is an overall scale factor. A naive mapping with at least four simulations in each direction of parameter space (as was sufficient for the current phenomenological nonprecessing models [2-4]) would imply that modeling these systems requires $4^{7} \sim O\left(10^{4}\right)$ numerical simulations.

In recent work, we identified an approximate mapping between inspiral waveforms from generic binaries, and those from a two-dimensional parameter space of nonprecessing binaries [5]. This approximation holds because precession has little effect on the inspiral rate, and so, precession effects approximately decouple from the overall inspiral, which can be described by a nonprecessing-binary model, neglecting the effect of breaking equatorial symmetry, which is responsible for large recoils [6]. Further, we proposed that, given a model for the precessional motion of a binary, we could construct an approximate waveform by twisting up the appropriate nonprecessing-binary waveform with the precessional motion. This technique was recently adopted to produce simple frequency-domain PN inspiral waveforms [7]. It was more recently suggested that this mapping also holds through merger and ringdown [8]. 
In this Letter, we take this idea further in two crucial ways. First, we use PN expressions for the precession angles to twist up a phenomenological model of nonprecessing-binary waveforms [4], which includes merger and ringdown. The inclusion of merger and ringdown provides the first frequency-domain inspiral-mergerringdown model of generic binaries. (Frequency-domain models are essential for both efficient GW searches and parameter estimation.) Our model uses the highest-order (closed-form) PN expressions available, and we also incorporate precession effects into the estimate of the final black-hole spin and the ringdown model.

Second, we make use of a single parameter that captures the basic precession phenomenology for generic binary configurations [9]. Our final model has only three intrinsic dimensionless physical parameters: the two parameters of our previous nonprecessing models (the mass ratio $q=m_{2} / m_{1} \geq 1$, and an effective inspiral spin, $\chi_{\text {eff }}$, which characterizes the rate of inspiral); plus one additional parameter, an effective precession spin, $\chi_{p}$. All other additional configuration parameters (the total mass, the binary's sky location, orientation, polarization, and initial orbital and precession phases), can be trivially included analytically. We describe this parametrization in more detail below; its effectiveness in capturing the phenomenology of the inspiral across the full parameter space is demonstrated in Ref. [9]. Our evaluation of its fidelity for $\mathrm{GW}$ applications when including merger and ringdown by comparison against hybrid PN-NR waveforms constitutes our core quantitative result.

The purpose of this model is to (a) facilitate the development of computationally efficient generic-binary searches, (b) provide a starting point to investigate the parameter-estimation possibilities (and limitations) of generic-binary observations in second-generation detectors, and their astrophysical implications, and (c) as a simple framework for the construction of more refined models calibrated to NR simulations. If the dominant parameter space of binary simulations can be reduced to three dimensions (mass ratio, effective inspiral spin, effective precession spin), it may be feasible to produce a sufficient number of NR waveforms $(\sim 100)$ to calibrate the model well before advanced detectors reach design sensitivity in 2018-2020 [10]. The model can be further refined, based on the results of these studies. As such, this model provides a practical road map to model generic binaries to meet the needs of GW astronomy over the next decade. This model has been included in the LAL data analysis software, to facilitate the development and testing of search and parameter estimation pipelines [11].

Model.-We start from the frequency-domain model (PhenomC) [4] of nonprecessing waveforms, because it includes the standard state-of-the-art inspiral phase. This model describes the $(\ell=2, m=|2|)$ modes of the waveform, with $h(f)=A(f) e^{i \psi(f)}$, where $A(f)$ and $\psi(f)$ are given in Ref. [4]. Based on the approximate mapping identified in Ref. [5], for a given generic binary, we start with the nonprecessing waveform given by the parameters $\left(M, \eta, \chi_{\text {eff }}\right)$, where $\eta=q /(1+q)^{2}$ and $\chi_{\text {eff }}=\left(m_{1} \chi_{1}+\right.$ $\left.m_{2} \chi_{2}\right) / M ; \chi_{1}$ and $\chi_{2}$ are the components of the dimensionless spins $\left(\chi_{i}=\boldsymbol{S}_{i} \cdot \hat{\boldsymbol{L}} / \mathrm{m}_{i}^{2}\right)$ projected along the Newtonian orbital angular momentum $\hat{\boldsymbol{L}}$. The direction of $\hat{\boldsymbol{J}}$ is approximately constant throughout the evolution, as angular-momentum loss via GWs is predominantly along $\hat{\boldsymbol{J}}$, with emission orthogonal to $\hat{\boldsymbol{J}}$ averaging out due to the precession of $\hat{\boldsymbol{L}}$ around $\hat{\boldsymbol{J}}$ [12]. Therefore, we assume that the final spin is in the same direction as $\hat{\boldsymbol{J}}$ through the inspiral, and update the PhenomC final spin magnitude estimate [13] to account for precession, using Ref. [14], with only one black hole spinning.

Then, we twist up the nonprecessing model; i.e., we approximate the $\ell=2$ modes of a precessing binary waveform in the time domain by rotating the dominant modes of the corresponding nonprecessing waveform $[5,15]$ as

$$
h_{2 m}^{P}(t)=e^{-i m \alpha} \sum_{\left|m^{\prime}\right|=2} e^{i m^{\prime} \epsilon} d_{m^{\prime}, m}^{2}(-l) h_{2, m^{\prime}}(t),
$$

where $d_{n m}^{\ell}$ denotes the Wigner $d$ matrices. The angles $\alpha$ and $l$ that enter our model are defined as the spherical angles parametrizing the unit Newtonian orbital angular momentum $\hat{\boldsymbol{L}}$ (see, for example, Fig. 1 in Ref. [9]) in an inertial frame with $\hat{z}=\hat{J}$. The third angle, defined from $\dot{\epsilon}=\dot{\alpha} \cos l$, parametrizes a rotation around $\hat{\boldsymbol{L}}$ [16]. During the inspiral phase, all of these angles vary slowly (on the precession time scale) with respect to the orbital time scale, which allows for a stationary-phase-approximation (SPA) transformation to the frequency domain (this fact has been exploited in work dating from Ref. [17], and was most recently used in Ref. [7]). Here, we use closed-form frequency-domain PN expressions for these angles (valid for systems with only one spin in the orbital plane) to twist the entire nonprecessing modes, formally continuing the SPA treatment through merger and ringdown. Although we do not expect these expressions, or the approximation of slowly varying precession angles, to be valid through merger and ringdown, in practice, we find that they mimic to reasonable accuracy the phenomenology of our PN-NR hybrids and lead to high fitting factors even for high masses. Our model consists entirely of closed-form analytic expressions, and the output is the two polarizations $h_{+, \times}^{P}\left(M f ; \eta, \chi_{\text {eff }}, \chi_{p}, \theta, \phi\right)$.

The inclination $l$ is simply the angle between the binary's total angular momentum, $\hat{\boldsymbol{J}}$, and orbital angular momentum, $\hat{\boldsymbol{L}}$, so that $\cos \iota=\hat{\boldsymbol{L}} \cdot \hat{\boldsymbol{J}}=\hat{\boldsymbol{L}} \cdot \boldsymbol{J} /|\boldsymbol{J}|$. In practice, we find that the accuracy in $l$, which enters only in amplitude factors for the contributions in (1), is not critical and that it is sufficient to include only nonspinning corrections in $\boldsymbol{J}$ beyond the total spin contribution at leading order. 
The precession angle $\alpha$ is computed using the expression for $\dot{\alpha}$ obtained in [18] [see Eqs. (4.10a) and (4.8)] by inserting the highest order (next-to-next-to-leading in spin-orbit) expressions available for the quantities entering the formula [19], PN reexpanding and averaging over the orientation of the spin in the orbital plane.

The spin parameters in our model are $\chi_{\text {eff }}$ and $\chi_{p}$. The effective inspiral spin $\chi_{\text {eff }}$ was defined earlier. The angle expressions $(\alpha, l)$, require some choice for the distribution of spins across the two black holes, and, for our implementation, we let $\chi_{1}=0$ and $\chi_{2}=\left(M / m_{2}\right) \chi_{\text {eff }}$; i.e., all of the spin is on the larger black hole. This choice performs well in the study in Ref. [9]. To ensure physical spins of $\chi \leq 1$ for each black hole, we could also choose $\chi_{1}=\chi_{2}=\chi_{\text {eff }}$. The implications of these choices for detection and parameter estimation will be explored in future work; in the cases we study here, we see that our model is likely to perform well for $\mathrm{GW}$ detection. The in-plane spin magnitude $\chi_{p}$ is associated with the larger black hole.

We expect our model to capture the basic phenomenology of generic two-spin systems, motivated by the following argument. For the effective precession spin, if $S_{1 \perp}$ and $S_{2 \perp}$ are the magnitudes of the projections of the two spins in the orbital plane, then, according to the $\mathrm{PN}$ precession equations $[12,20]$, the precession rate at leading order will be proportional to $\left(A_{1} S_{1 \perp}+A_{2} S_{2 \perp}\right)$ when the vectors $S_{1 \perp}$ and $\boldsymbol{S}_{2 \perp}$ are parallel, and by $\left(A_{1} S_{1 \perp}-A_{2} S_{2 \perp}\right)$ when they point in opposite directions, where $A_{i}=2+\left(3 m_{3-i}\right) /\left(2 m_{i}\right)$. During the inspiral, to first approximation, the average precession rate for non-equal-mass systems is simply the maximum of these two spin contributions, and we can define $S_{p}=\max \left(A_{1} S_{1 \perp}, A_{2} S_{2 \perp}\right) / A_{2}$, and expect that applying an in-plane spin of $\chi_{p}=S_{p} / m_{2}^{2}$ to the larger black hole will mimic the main precession effects of the full two-spin system. In equal-mass systems a double-spin configuration is indistinguishable from a single-spin system if we neglect spin-spin contributions; this changes the interpretation of $\chi_{p}$, but the use of two spin parameters is now automatically valid. (The third spin-vector component corresponds to the initial precession angle of the system, which is included as an overall complex factor in the model.) Full generic two-spin waveforms will typically exhibit additional small oscillations in the precession angles (see, e.g., Fig. 4 of Ref. [21], and Ref. [9]), but we do not expect these effects to be detectable in most GW observations. These two parameters, $\chi_{\text {eff }}$ and $\chi_{p}$, can be mapped to a range of physically allowable individual black-hole spins.

Results. - The most reliable way to test our model is to compare against hybrid PN (inspiral) and NR (mergerringdown) waveforms. But to do that across the full genericbinary parameter space would require the same number of waveforms as needed to construct a seven-dimensional generic model, which is the computationally prohibitive task that we wished to avoid in the first place. In practice, all we can do is identify what we expect to be challenging points in the parameter space. In this work, we restrict ourselves to binaries with mass ratios $q \leq 3$, because that is the mass ratio to which the underlying PhenomC model was calibrated to spinning-binary waveforms. We construct four hybrids at mass ratios 2 and 3, for a variety of spin choices. The numerical simulations were produced with the BAM code [22], and hybrids were constructed by the method described in Ref. [5], and also in the inertial frame of the NR waveforms, for comparison. The comparison configurations are chosen to include strong precession (a $q=3$ case where the larger black hole has a spin of $\chi_{2}=0.75$ in the orbital plane), a double-spin $q=2$ case, where the small black hole has spin 0.5 , and the larger black hole spin 0.75 , both in the orbital plane, which tests our assumption that we can consider only a weighted average of the spins when constructing $\chi_{p}$. We also consider a double-spin $q=3$ case where $\chi_{\text {eff }}=-0.5$ and $\chi_{p}=0.6$, and another where $\chi_{\text {eff }}=-0.125$ and $\chi_{p}=0.75$; the purpose here was to test the model with nonzero $\chi_{\text {eff }}$. The NR waveforms include between 10 and $14 \mathrm{GW}$ cycles before merger, and the PN part consists of $\sim 200$ cycles. For all waveforms we considered, there are approximately three premerger precession cycles, since the precession rate depends only weakly on the mass ratio.

As is standard in GW analysis, we calculate the noiseweighted inner product between our source waveform (in this case, the hybrid), and a model (either the original nonprecessing PhenomC model, or our new precessing PhenomP model). We use the current expectation for the design sensitivity of advanced LIGO [23], with a lowfrequency cutoff of $20 \mathrm{~Hz}$. This inner product is maximized with respect to the parameters of the model, including the physical parameters and the binary orientation and polarization. This optimized inner product is called the "fitting

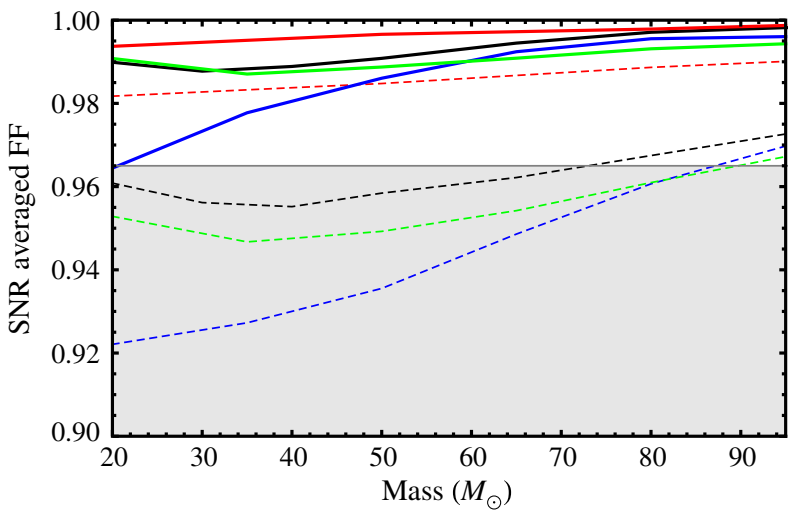

FIG. 1 (color online). Fitting factors (FF) between PhenomP (solid lines) and PhenomC (dashed lines), averaged over binary orientations, as described in the text. Each color is one case: $q=3, \chi_{p}=0.75$ (black); $q=2$ double spin (red); $q=3$, $\chi_{\text {eff }}=-0.5$ (green); $q=3, \chi_{\text {eff }}=-0.125$ (blue). We see that in all cases PhenomP meets the 0.965 threshold for detection accuracy. Above $100 M_{\odot}$, all of the curves are above 0.965 . 
factor"; its value indicates how well the signal can be found in detector data, and the bias between the best-fit model parameters, and the true source parameters, give us an indication of the errors in a GW measurement. We have computed fitting factors using PhenomC and PhenomP for total source masses between 20 and $200 M_{\odot}$ and as functions of binary orientations.

Figure 1 shows the fitting factor averaged across binary orientations, appropriately weighted with the signal-tonoise ratio to give an indication of the proportion of signals that would be detected. The standard requirement for GW searches is that the fitting factor be above 0.965 , corresponding to a loss of no more than $10 \%$ of sources in a search (disregarding additional loss due to a discrete template bank). We see that, in all cases, the PhenomP model achieves average fitting factors above this threshold. The PhenomC model is acceptable only at high masses.

The fitting factors are highest for near-optimal orientations, where the total angular momentum is aligned with
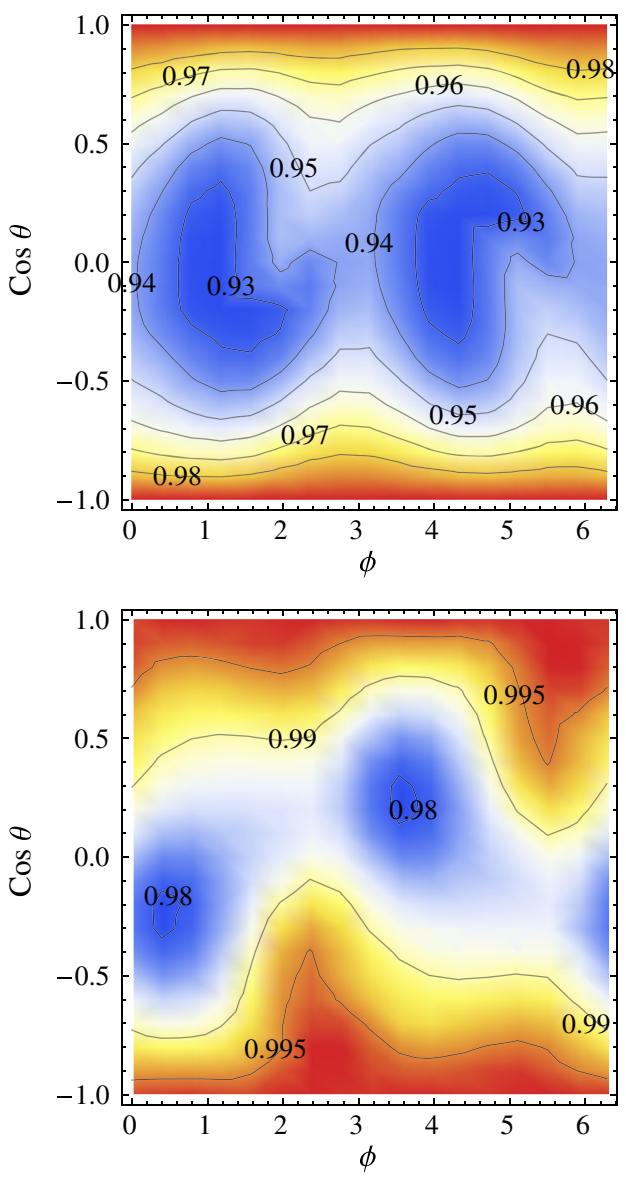

FIG. 2 (color online). Fitting factors (FF) between a $q=3$ highly precessing binary, and the nonprecessing PhenomC and precessing PhenomP models, as a function of binary orientation angles $(\theta, \phi)$; at $\theta=0$, an observer is oriented with the binary's total angular momentum. FF $<0.965$ for many orientations with PhenomC, while for PhenomP it is well above 0.965 for all orientations. See text for further details. the detector, and from which the precession has only a small effect on the signal. As an example, Fig. 2 shows results for the $q=3$ high precession configuration at $50 M_{\odot}$, which proved to be the most challenging configuration with no parallel spin component. Results are similar for lower masses, while for higher masses fitting factors improve at the expense of parameter accuracy. For the case with large negative $\chi_{\text {eff }}$ we found that for some orientations at masses below $60 M_{\odot}$, the fitting factor was below the 0.965 threshold. This is because of differences in the PN inspiral approximants used for the hybrid and for the model; these effects for large antialigned spins have been observed in the past [24], and are independent of our modeling procedure. We leave a full study of parameter biases to future work, but our results suggest that a measurement of $\chi_{p}$ reliably identifies precession.

Discussion.-We have presented the first frequencydomain inspiral-merger-ringdown model for the GW signal from precessing-black-hole binaries. Incorporating a series of insights from our previous work, our model is constructed by a straightforward transformation of a nonprecessingbinary model, in this case PhenomC; in practice, any workable nonprecessing model could be used instead. The current model did not require any precessing-binary numerical simulations in its construction, although, in the future, we plan to use extensive simulations to refine the model, based on tests of the model's accuracy for GW searches and parameter estimation. Finally, we are able to model the essential phenomenology of the sevendimensional parameter space of binary configurations with a model that requires only three key physical parameters. This will simplify the model's incorporation into search and parameter estimation pipelines, as well as making tractable the problem of producing enough numerical simulations to produce a model of sufficient accuracy for GW astronomy with advanced detectors. It is not clear whether a precessing inspiral-merger-ringdown model will be necessary for GW searches, or the level of accuracy that is required for parameter estimation studies, but these questions can only be answered once a (reasonably fast to evaluate) model exists, and we have provided one.

Our ability to model generic waveforms with only two spin parameters implies strong degeneracies that will make it difficult to identify the individual black-hole spins, in particular, the spin of the smaller black hole. This may well be the reality of GW observations with second-generation detectors, for which $80 \%$ of signals will be at signal-tonoise ratios between 10 and 20, in which the subtle doublespin effects on the waveform may be difficult to identify. These are important issues that deserve further attention in future work.

The current model is valid only in the region of parameter space for which PhenomC was calibrated $\left(q \leq 4,\left|\chi_{\text {eff }}\right| \leq 0.85\right)$. More challenging precession cases are expected at higher mass ratios and spins (e.g., 
transitional precession), and the ability of our prescription to model those configurations will need to be tested when refined frequency-domain nonprecessing-binary models become available.

We thank P. Ajith and S. Fairhurst for useful discussions. P. S. was supported by a DOC-fFORTE-Fellowship of the Austrian Academy of Sciences and was also partially supported by the STFC. M. H. was supported by STFC Grants No. ST/H008438/1 and No. ST/I001085/1, and F. O. and M. P. by Grant No. ST/I001085/1. A. B. and S. H. were supported by the Spanish MIMECO Grants No. FPA2010-16495 and No. CSD2009-00064, European Union FEDER funds, and Conselleria d'Economia i Competitivitat del Govern de les Illes Balears. G. P. was supported by an STFC doctoral training grant. L. H. was supported by the Conseil Général de l'Essonne. Numerical simulations were carried out at ARCCA in Cardiff, the UK DiRAC Data centric cluster, MareNostrum at Barcelona Supercomputing Center-Centro Nacional de Supercomputación, and on the PRACE clusters Hermit, Curie, and SuperMUC.

[1] B. Sathyaprakash and B. F. Schutz, Living Rev. Relativity 12, 1 (2009).

[2] P. Ajith et al., Phys. Rev. D 77, 104017 (2008).

[3] P. Ajith et al., Phys. Rev. Lett. 106, 241101 (2011).

[4] L. Santamaría et al., Phys. Rev. D 82, 064016 (2010).

[5] P. Schmidt, M. Hannam, and S. Husa, Phys. Rev. D 86, 104063 (2012).

[6] B. Brügmann, J. A. González, M. Hannam, S. Husa, and U. Sperhake, Phys. Rev. D 77, 124047 (2008).
[7] A. Lundgren and R. O'Shaughnessy, Phys. Rev. D 89, 044021 (2014).

[8] L. Pekowsky, R. O'Shaughnessy, J. Healy, and D. Shoemaker, Phys. Rev. D 88, 024040 (2013).

[9] P. Schmidt, F. Ohme, and M. Hannam, arXiv:1408.1810.

[10] J. Aasi et al. (LIGO Scientific Collaboration and Virgo Collaboration), arXiv:1304.0670.

[11] LSC Algorithm Library (LAL), http://www.lsc-group.phys .uwm.edu/lal.

[12] T. A. Apostolatos, C. Cutler, G. J. Sussman, and K. S. Thorne, Phys. Rev. D 49, 6274 (1994).

[13] E. Berti, V. Cardoso, and C. M. Will, Phys. Rev. D 73, 064030 (2006).

[14] E. Barausse and L. Rezzolla, Astrophys. J. 704, L40 (2009).

[15] P. Schmidt, M. Hannam, S. Husa, and P. Ajith, Phys. Rev. D 84, 024046 (2011).

[16] M. Boyle, R. Owen, and H. P. Pfeiffer, Phys. Rev. D 84, 124011 (2011).

[17] T. A. Apostolatos, Phys. Rev. D 54, 2421 (1996).

[18] L. Blanchet, A. Buonanno, and G. Faye, Phys. Rev. D 84, 064041 (2011).

[19] A. Bohe, S. Marsat, G. Faye, and L. Blanchet, Classical Quantum Gravity 30, 075017 (2013).

[20] L. E. Kidder, Phys. Rev. D 52, 821 (1995).

[21] A. Buonanno, Y.-b. Chen, Y. Pan, and M. Vallisneri, Phys. Rev. D 70, 104003 (2004).

[22] B. Brügmann, J. A. González, M. Hannam, S. Husa, U. Sperhake, and W. Tichy, Phys. Rev. D 77, 024027 (2008).

[23] LIGO Scientific Collaboration, Report No. LIGOT0900288-v3, LIGO Project, 2009, https://dcc.ligo.org/ LIGO-T0900288-v3/public.

[24] A. H. Nitz, A. Lundgren, D. A. Brown, E. Ochsner, D. Keppel, and D. Keppel, and Ian Harry, Phys. Rev. D 88, 124039 (2013). 